\title{
Triple Representation Theorem for homogeneous effect algebras
}

\author{
Josef Niederle and Jan Paseka \\ Department of Mathematics and Statistics \\ Faculty of Science, Masaryk University \\ Kotlářská 2, CZ-611 37 Brno, Czech Republic \\ E-mail: niederle,paseka@math.muni.cz
}

\begin{abstract}
Dedicated to Ivo G. Rosenberg on the occasion of his 77th birthday
in appreciation of his contributions to the fields of multiple-valued logic
\end{abstract}

\begin{abstract}
The aim of our paper is to prove the Triple Representation Theorem, which was established by Jenča in the setting of complete lattice effect algebras, for a special class of homogeneous effect algebras, namely TRTeffect algebras. This class includes complete lattice effect algebras, sharply dominating Archimedean atomic lattice effect algebras and homogeneous orthocomplete effect algebras.
\end{abstract}

Keywords-Homogeneous effect algebra, TRT-effect algebra, orthocomplete effect algebra, lattice effect algebra, MV-algebra, block, center, atom, sharp element, meager element, sharply dominating effect algebra.

\section{INTRODUCTION}

Two equivalent quantum structures, D-posets and effect algebras were introduced in the nineties of the twentieth century. These were considered as "unsharp" generalizations of the structures which arise in quantum mechanics, in particular, of orthomodular lattices and MV-algebras. Effect algebras aim to describe "unsharp" event structures in quantum mechanics in the language of algebra.

Effect algebras are fundamental in investigations of fuzzy probability theory too. In the fuzzy probability frame, the elements of an effect algebra represent fuzzy events which are used to construct fuzzy random variables.

The aim of our paper is to prove the Triple Representation Theorem, which was established by Jenča in [9] in the setting of complete lattice effect algebras, for a special class of homogeneous effect algebras, namely TRT-effect algebras. This class includes complete lattice effect algebras, sharply dominating Archimedean atomic lattice effect algebras (see [12]) and homogeneous orthocomplete effect algebras (see [13]).

\section{PRELIMINARIES AND BASIC FACTS}

Effect algebras were introduced by Foulis and Bennett (see [3]) for modelling unsharp measurements in a Hilbert space. In this case the set $\mathcal{E}(H)$ of effects is the set of all self-adjoint operators $A$ on a Hilbert space $H$ between the null operator 0 and the identity operator 1 and endowed with the partial operation + defined iff $A+B$ is in $\mathcal{E}(H)$, where + is the usual operator sum.

In general form, an effect algebra is in fact a partial algebra with one partial binary operation and two unary operations satisfying the following axioms due to Foulis and Bennett.

Definition I.1. [3] A partial algebra $(E ; \oplus, 0,1)$ is called an effect algebra if 0, 1 are two distinct elements, called the zero and the unit element, and $\oplus$ is a partially defined binary operation called the orthosummation on $E$ which satisfies the following conditions for any $x, y, z \in E$ :

(Ei) $\quad x \oplus y=y \oplus x$ if $x \oplus y$ is defined,

(Eii) $\quad(x \oplus y) \oplus z=x \oplus(y \oplus z)$ if one side is defined,

(Eiii) for every $x \in E$ there exists a unique $y \in$ $E$ such that $x \oplus y=1$ (we put $x^{\prime}=y$ ),

(Eiv) if $1 \oplus x$ is defined then $x=0$. 
$(E ; \oplus, 0,1)$ is called an orthoalgebra if $x \oplus x$ exists implies that $x=0$ (see [4]).

We often denote the effect algebra $(E ; \oplus, 0,1)$ briefly by $E$. On every effect algebra $E$ a partial order $\leqslant$ and a partial binary operation $\ominus$ can be introduced as follows:

$$
\begin{gathered}
x \leqslant y \text { and } y \ominus x=z \text { iff } x \oplus z \\
\text { is defined and } x \oplus z=y .
\end{gathered}
$$

If $E$ with the defined partial order is a lattice (a complete lattice) then $(E ; \oplus, 0,1)$ is called a lattice effect algebra (a complete lattice effect algebra).

Mappings from one effect algebra to another one that preserve units and orthosums are called morphisms of effect algebras, and bijective morphisms of effect algebras having inverses that are morphisms of effect algebras are called isomorphisms of effect algebras.

Definition I.2. Let $E$ be an effect algebra. Then $Q \subseteq E$ is called a sub-effect algebra of $E$ if

(i) $1 \in Q$

(ii) if out of elements $x, y, z \in E$ with $x \oplus y=z$ two are in $Q$, then $x, y, z \in Q$.

Note that a sub-effect algebra $Q$ of an effect algebra $E$ with inherited operation $\oplus$ is an effect algebra in its own right.

Definition I.3. (1): A generalized effect algebra ( $E$; $\oplus, 0)$ is a set $E$ with element $0 \in E$ and partial binary operation $\oplus$ satisfying, for any $x, y, z \in E$, the conditions

(GE1) $x \oplus y=y \oplus x$ if one side is defined,

(GE2) $(x \oplus y) \oplus z=x \oplus(y \oplus z)$ if one side is defined

(GE3) if $x \oplus y=x \oplus z$ then $y=z$,

(GE4) if $x \oplus y=0$ then $x=y=0$,

(GE5) $x \oplus 0=x$ for all $x \in E$.

(2) A binary relation $\leqslant$ (being a partial order) and a partial binary operation $\ominus$ on $E$ can be defined by:

$$
\begin{gathered}
x \leqslant y \text { and } y \ominus x=z \text { iff } x \oplus z \\
\text { is defined and } x \oplus z=y .
\end{gathered}
$$

(3) A nonempty subset $Q \subseteq E$ is called a subgeneralized effect algebra of $E$ if out of elements $x, y, z \in E$ with $x \oplus y=z$ at least two are in $Q$ then $x, y, z \in Q$.
Every sub-generalized effect algebra of $E$ is a generalized effect algebra in its own right. Every effect algebra is a generalized effect algebra.

For an element $x$ of a generalized effect algebra $E$ we write $\operatorname{ord}(x)=\infty$ if $n x=x \oplus x \oplus \cdots \oplus$ $x$ (n-times) exists for every positive integer $n$ and we write $\operatorname{ord}(x)=n_{x}$ if $n_{x}$ is the greatest positive integer such that $n_{x} x$ exists in $E$. A generalized effect algebra $E$ is Archimedean if $\operatorname{ord}(x)<\infty$ for all $x \in E$.

A minimal nonzero element of a generalized effect algebra $E$ is called an atom and $E$ is called atomic if below every nonzero element of $E$ there is an atom.

Definition I.4. We say that a finite system $F=$ $\left(x_{k}\right)_{k=1}^{n}$ of not necessarily different elements of a generalized effect algebra $E$ is orthogonal if $x_{1} \oplus$ $x_{2} \oplus \cdots \oplus x_{n}$ (written $\bigoplus_{k=1}^{n} x_{k}$ or $\bigoplus F$ ) exists in $E$. Here we define $x_{1} \oplus x_{2} \oplus \cdots \oplus x_{n}=\left(x_{1} \oplus x_{2} \oplus\right.$ $\left.\cdots \oplus x_{n-1}\right) \oplus x_{n}$ supposing that $\bigoplus_{k=1} x_{k}$ is defined and $\left(\bigoplus_{k=1}^{n-1} x_{k}\right) \oplus x_{n}$ exists. We also define $\bigoplus \emptyset=0$. An arbitrary system $G=\left(x_{\kappa}\right)_{\kappa \in H}$ of not necessarily different elements of $E$ is called orthogonal if $\bigoplus K$ exists for every finite $K \subseteq G$. We say that for an orthogonal system $G=\left(x_{\kappa}\right)_{\kappa \in H}$ the element $\bigoplus G$ exists iff $\bigvee\{\bigoplus K \mid K \subseteq G$ is finite $\}$ exists in $E$ and then we put $\bigoplus G=\bigvee\{\bigoplus K \mid K \subseteq G$ is finite $\}$. We say that $\bigoplus G$ is the orthogonal sum of $G$ and $G$ is orthosummable. (Here we write $G_{1} \subseteq G$ iff there is $H_{1} \subseteq H$ such that $\left.G_{1}=\left(x_{\kappa}\right)_{\kappa \in H_{1}}\right)$. We denote $G^{\oplus}:=\{\bigoplus K \mid K \subseteq G$ is finite $\} . G$ is called bounded if there is an upper bound of $G^{\oplus}$.

A generalized effect algebra $E$ is called orthocomplete if every bounded orthogonal system is orthosummable.

A generalized effect algebra $E$ has the maximality property if $\{u, v\}$ has a maximal lower bound $w$ for every $u, v \in E$.

Definition I.5. An element $x$ of an effect algebra $E$ is called

(i) sharp if $x \wedge x^{\prime}=0$. The set $\mathrm{S}(E)=\{x \in$ $\left.E \mid x \wedge x^{\prime}=0\right\}$ is called the set of all sharp elements of $E$ (see [6]). 
(ii) principal, if $y \oplus z \leq x$ for every $y, z \in E$ such that $y, z \leq x$ and $y \oplus z$ exists.

(iii) central, if $x$ and $x^{\prime}$ are principal and, for every $y \in E$ there are $y_{1}, y_{2} \in E$ such that $y_{1} \leq$ $x, y_{2} \leq x^{\prime}$, and $y=y_{1} \oplus y_{2}$ (see [5]). The center $\mathrm{C}(E)$ of $E$ is the set of all central elements of E.

If $x \in E$ is a principal element, then $x$ is sharp and the interval $[0, x]$ is an effect algebra with the greatest element $x$ and the partial operation given by restriction of $\oplus$ to $[0, x]$.

Statement I.6. [5, Theorem 5.4] The center $\mathrm{C}(E)$ of an effect algebra $E$ is a sub-effect algebra of $E$ and forms a Boolean algebra. For every central element $x$ of $E, y=(y \wedge x) \oplus\left(y \wedge x^{\prime}\right)$ for all $y \in E$. If $x, y \in \mathrm{C}(E)$ are orthogonal, we have $x \vee y=x \oplus y$ and $x \wedge y=0$.

Statement I.7. [10, Lemma 3.1.] Let $E$ be an effect algebra, $x, y \in E$ and $c, d \in \mathrm{C}(E)$. Then:

(i) If $x \oplus y$ exists then $c \wedge(x \oplus y)=(c \wedge x) \oplus(c \wedge y)$.

(ii) If $c \oplus d$ exists then $x \wedge(c \oplus d)=(x \wedge c) \oplus(x \wedge d)$.

Definition I.8. ( [8], [9] ) A subset $M$ of a generalized effect algebra $E$ is called internally compatible (compatible) if for every finite subset $M_{F}$ of $M$ there is a finite orthogonal family $\left(x_{1}, \ldots, x_{n}\right)$ of elements from $M(E)$ such that for every $m \in M_{F}$ there is a set $A_{F} \subseteq\{1, \ldots, n\}$ with $m=\bigoplus_{i \in A_{F}} x_{i}$. If $\{x, y\}$ is a compatible set, we write $x \leftrightarrow y$ (see [9], [11]).

\section{HomogeneOUS EFFECT ALGEBRAS}

Definition II.1. An effect algebra $E$ satisfies the Riesz decomposition property (or RDP) if, for all $u, v_{1}, v_{2} \in E$ such that $u \leq v_{1} \oplus v_{2}$, there are $u_{1}, u_{2}$ such that $u_{1} \leq v_{1}, u_{2} \leq v_{2}$ and $u=u_{1} \oplus u_{2}$.

A lattice effect algebra in which RDP holds is called an $M V$-effect algebra.

An effect algebra $E$ is called homogeneous if, for all $u, v_{1}, v_{2} \in E$ such that $u \leq v_{1} \oplus v_{2} \leq u^{\prime}$, there are $u_{1}, u_{2}$ such that $u_{1} \leq v_{1}, u_{2} \leq v_{2}$ and $u=u_{1} \oplus u_{2}$ (see [8]).

Statement II.2. [9, Proposition 2]

(i) Every orthoalgebra is homogeneous.

(ii) Every lattice effect algebra is homogeneous. (iii) An effect algebra $E$ has the Riesz decomposition property if and only if $E$ is homogeneous and compatible.

Let $E$ be a homogeneous effect algebra.

(iv) A subset $B$ of $E$ is a maximal sub-effect algebra of $E$ with the Riesz decomposition property (such $B$ is called a block of $E$ ) if and only if $B$ is a maximal internally compatible subset of $E$ containing 1.

(v) Every finite compatible subset of $E$ is a subset of some block. This implies that every homogeneous effect algebra is a union of its blocks.

(vi) $\mathrm{S}(E)$ is a sub-effect algebra of $E$.

(vii) For every block $B, \mathrm{C}(B)=\mathrm{S}(E) \cap B$.

(viii) Let $x \in B$, where $B$ is a block of $E$. Then $\left\{y \in E \mid y \leq x\right.$ and $\left.y \leq x^{\prime}\right\} \subseteq B$.

An important class of effect algebras was introduced by Gudder in [6] and [7]. Fundamental example is the standard Hilbert spaces effect algebra $\mathcal{E}(\mathcal{H})$.

For an element $x$ of an effect algebra $E$ we denote

$$
\begin{array}{ll}
\widetilde{x}=\bigvee_{E}\{s \in \mathrm{S}(E) \mid s \leq x\} & \begin{array}{l}
\text { if it exists and } \\
\text { belongs to } \mathrm{S}(E),
\end{array} \\
\widehat{x}=\bigwedge_{E}\{s \in \mathrm{S}(E) \mid s \geq x\} & \begin{array}{l}
\text { if it exists and } \\
\text { belongs to } \mathrm{S}(E) .
\end{array}
\end{array}
$$

Definition II.3. ( [6], [7]) An effect algebra $(E ; \oplus, 0,1)$ is called sharply dominating if for every $x \in E$ there exists $\widehat{x}$, the smallest sharp element such that $x \leq \widehat{x}$. That is $\widehat{x} \in \mathbf{S}(E)$ and if $y \in \mathbf{S}(E)$ satisfies $x \leq y$ then $\widehat{x} \leq y$.

Recall that evidently an effect algebra $E$ is sharply dominating iff for every $x \in E$ there exists $\widetilde{x} \in \mathbf{S}(E)$ such that $\widetilde{x} \leq x$ and if $u \in \mathbf{S}(E)$ satisfies $u \leq x$ then $u \leq \widetilde{x}$.

In what follows set (see [9], [14])

$$
\begin{aligned}
\mathrm{M}(E)=\{x \in E \mid & \text { if } v \in \mathrm{S}(E) \text { satisfies } \\
v & \leq x \text { then } v=0\} .
\end{aligned}
$$

An element $x \in \mathbf{M}(E)$ is called meager. Moreover, $x \in \mathbf{M}(E)$ iff $\widetilde{x}=0$. Recall that $x \in \mathbf{M}(E)$, $y \in E, y \leq x$ implies $y \in \mathbf{M}(E)$ and $x \ominus y \in \mathbf{M}(E)$. Moreover, we have a map $h: \mathrm{S}(E) \rightarrow 2^{\mathrm{M}(E)}$ that is given by $h(s)=\{x \in \mathbf{M}(E) \mid x \leq s\}$.

Recall that $\mathbf{M}(E)$ equipped with a partial operation $\oplus_{\mathbf{M}(E)}$ which is defined, for all $x, y \in \mathbf{M}(E)$, by $x \oplus_{\mathrm{M}(E)} y$ exists if and only if $x \oplus_{E} y$ exists and 
$x \oplus_{E} y \in \mathbf{M}(E)$ in which case $x \oplus_{\mathbf{M}(E)} y=x \oplus_{E} y$ is a generalized effect algebra.

Statement II.4. [12, Lemma 2.4] Let $E$ be an effect algebra in which $\mathrm{S}(E)$ is a sub-effect algebra of $E$ and let $x \in \mathrm{M}(E)$ such that $\widehat{x}$ exists. Then

(i) $\widehat{x} \ominus x \in \mathbf{M}(E)$.

(ii) If $y \in \mathrm{M}(E)$ such that $x \oplus y$ exists and $x \oplus y=z \in \mathrm{S}(E)$ then $\widehat{x}=z$.

Statement II.5. [12, Lemma 2.5] Let $E$ be an effect algebra in which $\mathrm{S}(E)$ is a sub-effect algebra of $E$ and let $x \in E$ such that $\widetilde{x}$ exists. Then $x \ominus \widetilde{x} \in \mathbf{M}(E)$ and $x=\widetilde{x} \oplus(x \ominus \widetilde{x})$ is the unique decomposition $x=x_{S} \oplus x_{M}$, where $x_{S} \in \mathrm{S}(E)$ and $x_{M} \in \mathbf{M}(E)$. Moreover, $x_{S} \wedge x_{M}=0$ and if $E$ is a lattice effect algebra then $x=x_{S} \vee x_{M}$.

As proved in [1], $\mathrm{S}(E)$ is always a sub-effect algebra in a sharply dominating effect algebra $E$.

Statement II.6. [9, Corollary 14], [13, Lemma 4.2] Let $E$ be an orthocomplete homogeneous effect algebra.

(i) $E$ is sharply dominating and, for every block $B$ of $E, x \in B$ implies that $[\widetilde{x}, x] \subseteq B$.

(ii) Let $x \in \mathbf{M}(E)$. Then $y=\widehat{x} \ominus x$ is the only element such that

a) $y \in \mathbf{M}(E)$ such that $\widehat{y}=\widehat{x}$.

b) $x \bigoplus_{\mathrm{M}(E)}\left(y \ominus_{\mathrm{M}(E)}(x \wedge y)\right)$ exists and $x \oplus_{\mathbf{M}(E)}\left(y \ominus_{\mathrm{M}(E)}(x \wedge y)\right) \in h(\widehat{x})$.

c) For all $z \in h(\widehat{x}), z \oplus_{\mathrm{M}(E)} x \in h(\widehat{x})$ if and only if $z \leq y$ and $\overline{y \ominus_{\mathbf{M}(E)} z}=\widehat{x}$.

\section{TRIPLE REPRESENTATION THEOREM FOR TRT-EFFECT ALGEBRAS}

In what follows $E$ will be always a homogeneous sharply dominating effect algebra such that, for every block $B$ of $E, x \in B$ implies that $[\widetilde{x}, x] \subseteq B$ and, for all $x \in \mathbf{M}(E)$ the element $y=\widehat{x} \ominus x$ is the only element such that

(i) $y \in \mathbf{M}(E)$ such that $\widehat{y}=\widehat{x}$.

(ii) $x \bigoplus_{\mathbf{M}(E)}\left(y \ominus_{\mathrm{M}(E)}(x \wedge y)\right)$ exists and $x \oplus_{\mathbf{M}(E)}$ $\left(y \ominus_{\mathbf{M}(E)}(x \wedge y)\right) \in h(\widehat{x})$.

(iii) For all $z \in h(\widehat{x}), z \oplus_{\mathrm{M}(E)} x \in h(\widehat{x})$ if and only if $z \leq y$ and $\overline{y \ominus_{\mathrm{M}(E)} z}=\widehat{x}$.

We will call such an effect algebra a TRT-effect algebra. In this case $\mathrm{S}(E)$ is a sub-effect algebra of $E$.
Since $E$ is sharply dominating we have that, for all $x \in \mathbf{M}(E)$,

$$
\begin{aligned}
\widehat{x} & =\bigwedge_{E}\{s \in \mathrm{S}(E) \mid x \in h(s)\} \\
& =\bigwedge_{\mathrm{S}(E)}\{s \in \mathrm{S}(E) \mid x \in h(s)\} .
\end{aligned}
$$

This gives us

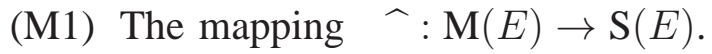

Note that, if $x \in \mathrm{M}(E), s \in \mathrm{S}(E)$ and $B$ is a block of $E$ such that $x, s \in B$ then from Statement I.6 we have that $x \wedge_{B} s \leq x$ exists. Assume that $g \in E, g \leq s$ and $g \leq x$. Then $g \in B$ and therefore $g \leq x \wedge_{B} s$. It follows that $x \wedge_{E} s=x \wedge_{B} s$. We always have the following partial map.

(M2) For every $s \in \mathrm{S}(E)$, a partial mapping $\pi_{s}$ : $\mathrm{M}(E) \rightarrow h(s)$ is given by $\pi_{s}(x)=x \wedge_{E} s$ whenever $\pi_{s}(x)$ is defined.

Therefore, if $x \leftrightarrow s$ then $x \wedge_{E} s$ exists and $\pi_{s}(x)$ is defined.

We also have the following map $R$ which is defined entirely in terms of the triple.

(M3) The mapping $R: \mathrm{M}(E) \rightarrow \mathrm{M}(E)$ given by $R(x)=\widehat{x} \ominus_{E} x$.

Let $x, y \in \mathbf{M}(E)$. Let us put $\mathcal{S}(x, y)=$ $\left\{z \in \mathrm{S}(E) \mid z=(z \wedge x) \oplus_{E}(z \wedge y)\right\}=$ $\left\{z \in \mathrm{S}(E) \mid \pi_{z}(x)\right.$ and $\pi_{z}(x)$ are defined, $z=$ $\overline{\pi_{z}(x)}$ and $\left.R\left(\pi_{z}(x)\right)=\pi_{z}(y)\right\}$.

(M4) The partial mapping $S: \mathrm{M}(E) \times \mathrm{M}(E) \rightarrow$ $\mathrm{S}(E)$ given by $S(x, y)$ is defined if and only if the set $\mathcal{S}(x, y)=\{z \in \mathrm{S}(E) \mid z \wedge x$ and $z \wedge$ $y$ exist, $\left.z=(z \wedge x) \oplus_{E}(z \wedge y)\right\}$ has a top element $z_{0} \in \mathcal{S}(x, y)$ in which case $S(x, y)=$ $z_{0}$.

Whether $S(x, y)$ is defined or not we are able to decide in terms of the triple. Since the eventual top element $z_{0}$ of $\mathcal{S}(x, y)$ is in $\mathbf{S}(E)$ our definition of $S(x, y)$ is correct.

Triple Representation Theorem The triple $\left(\left(\mathbf{S}(E), \oplus_{\mathbf{S}(E)}\right),\left(\mathbf{M}(E), \oplus_{\mathbf{M}(E)}\right), h\right)$ characterizes $E$ up to isomorphism within the class of all TRT-effect algebras.

We have to construct an isomorphic copy of the original effect algebra $E$ from the triple $(\mathrm{S}(E), \mathbf{M}(E), h)$. 
Lemma III.1. Let $E$ be a TRT-effect algebra, $x, y \in$ $\mathrm{M}(E)$. Then $x \oplus_{E} y$ exists in $E$ iff $S(x, y)$ is defined in terms of the triple $(\mathrm{S}(E), \mathrm{M}(E), h)$ and $\left(x \ominus_{\mathrm{M}(E)}\right.$ $(S(x, y) \wedge x)) \oplus_{\mathrm{M}(E)}\left(y \ominus_{\mathrm{M}(E)}(S(x, y) \wedge y)\right)$ exists in $\mathrm{M}(E)$ such that $\left(x \ominus_{\mathrm{M}(E)}(S(x, y) \wedge x)\right) \oplus_{\mathrm{M}(E)}$ $\left(y \ominus_{\mathrm{M}(E)}(S(x, y) \wedge y)\right) \in h\left(S(x, y)^{\prime}\right)$. Moreover, in that case

$$
\begin{aligned}
x \oplus_{E} y=S(x, y) & \oplus_{E}\left(\left(x \ominus_{\mathbf{M}(E)}(S(x, y) \wedge x)\right)\right. \\
& \left.\bigoplus_{\mathbf{M}(E)}\left(y \ominus_{\mathbf{M}(E)}(S(x, y) \wedge y)\right)\right) .
\end{aligned}
$$

Proof: Assume first that $x \oplus_{E} y$ exists in $E$ and let us put $z=x \oplus_{E} y$. Since $E$ is sharply dominating we have that $z=z_{S} \oplus_{E} z_{M}$ such that $z_{S} \in \mathbf{S}(E)$ and $z_{M} \in \mathbf{M}(E)$. Since $x \leftrightarrow y$ by Statement Щ.2. (e) there is a block $B$ of $E$ such that $x, y, z \in B$. Moreover, $E$ being a TRT-effect algebra yields that $\left[z_{S}, z\right] \subseteq B$ and we obtain that $z_{S}, z_{M} \in B$. Therefore $z_{S} \in \mathrm{C}(B)$ and by Statement 1.7, (i) we have that $z_{S}=z_{S} \wedge\left(x \oplus_{E} y\right)=z_{S} \wedge\left(x \oplus_{B} y\right)=$ $\left(z_{S} \wedge_{B} x\right) \oplus_{B}\left(z_{S} \wedge_{B} y\right)=\left(z_{S} \wedge x\right) \oplus_{E}\left(z_{S} \wedge y\right)$. Hence $z_{S} \in \mathcal{S}(x, y)$. Now, assume that $u \in \mathcal{S}(x, y)$. Then $u=(u \wedge x) \oplus_{E}(u \wedge y) \leq x \oplus_{E} y$. Since $u \in \mathbf{S}(E)$ we have that $u \leq z_{S}$, i.e., $z_{S}$ is the top element of $\mathcal{S}(x, y)$. Moreover, we have

$$
\begin{aligned}
& z_{S} \oplus_{E} z_{M}=x \oplus_{E} y \\
&=\left(((S(x, y) \wedge x)) \oplus_{E}\left(x \ominus_{E}(S(x, y) \wedge x)\right)\right) \\
& \oplus_{E}\left(((S(x, y) \wedge y)) \oplus_{E}\left(y \ominus_{E}(S(x, y) \wedge y)\right)\right) \\
&= S(x, y) \oplus_{E}\left(\left(x \ominus_{\mathrm{M}(E)}(S(x, y) \wedge x)\right)\right. \\
&\left.\oplus_{E}\left(y \ominus_{\mathrm{M}(E)}(S(x, y) \wedge y)\right)\right) .
\end{aligned}
$$

It follows that $z_{M}=\left(x \ominus_{\mathbf{M}(E)}(S(x, y) \wedge x)\right) \oplus_{E}$ $\left(y \ominus_{\mathrm{M}(E)}(S(x, y) \wedge y)\right)$ and evidently $z_{M} \in h\left(z_{S}^{\prime}\right)$.

Conversely, let us assume that $S(x, y)$ is defined in terms of $(\mathrm{S}(E), \mathbf{M}(E), h),\left(x \ominus_{\mathbf{M}(E)}(S(x, y) \wedge\right.$ $x)) \oplus_{\mathbf{M}(E)}\left(y \ominus_{\mathbf{M}(E)}(S(x, y) \wedge y)\right)$ exists in $\mathbf{M}(E)$ and $\left(x \ominus_{\mathbf{M}(E)}(S(x, y) \wedge x)\right) \oplus_{\mathbf{M}(E)}\left(y \ominus_{\mathbf{M}(E)}(S(x, y) \wedge\right.$ $y)) \in h\left(S(x, y)^{\prime}\right)$. Then $\left(x \ominus_{\mathrm{M}(E)}(S(x, y) \wedge\right.$

$$
\begin{aligned}
x)) \oplus_{\mathbf{M}(E)}\left(y \ominus_{\mathbf{M}(E)}(S(x, y) \wedge y)\right) \leq S(x, y)^{\prime}, \text { i.e., } \\
z=\quad S(x, y) \oplus_{E}\left(\left(x \ominus_{\mathbf{M}(E)}(S(x, y) \wedge x)\right)\right. \\
\left.\oplus_{\mathbf{M}(E)}\left(y \ominus_{\mathbf{M}(E)}(S(x, y) \wedge y)\right)\right) \\
=\quad\left((S(x, y) \wedge x) \oplus_{E}(S(x, y) \wedge y)\right) \oplus_{E} \\
\left(\left(x \ominus_{E}(S(x, y) \wedge x)\right) \oplus_{E}\right. \\
\left.\left(y \ominus_{E}(S(x, y) \wedge y)\right)\right)=x \oplus_{E} y
\end{aligned}
$$

is defined.

Theorem III.2. Let $E$ be a TRT-effect algebra. Let $\mathrm{T}(E)$ be a subset of $\mathrm{S}(E) \times \mathrm{M}(E)$ given by

$\mathrm{T}(E)=\left\{\left(z_{S}, z_{M}\right) \in \mathbf{S}(E) \times \mathbf{M}(E) \mid z_{M} \in h\left(z_{S}^{\prime}\right)\right\}$.

Equip $\mathrm{T}(E)$ with a partial binary operation $\oplus_{\mathrm{T}(E)}$ with $\left(x_{S}, x_{M}\right) \oplus_{\mathrm{T}(E)}\left(y_{S}, y_{M}\right)$ is defined if and only if

(i) $S\left(x_{M}, y_{M}\right)$ is defined,

(ii) $z_{S}=x_{S} \oplus_{\mathrm{S}(E)} y_{S} \oplus_{\mathrm{S}(E)} S\left(x_{M}, y_{M}\right)$ is defined,

(iii) $z_{M}=\left(x_{M} \ominus_{\mathrm{M}(E)}\left(S\left(x_{M}, y_{M}\right) \wedge x_{M}\right)\right) \oplus_{\mathrm{M}(E)}$ $\left(y_{M} \ominus_{\mathrm{M}(E)}\left(S\left(x_{M}, y_{M}\right) \wedge y_{M}\right)\right)$ is defined,

(iv) $z_{M} \in h\left(z_{S}^{\prime}\right)$.

In this case $\left(z_{S}, z_{M}\right)=\left(x_{S}, x_{M}\right) \oplus_{\mathrm{T}(E)}\left(y_{S}, y_{M}\right)$. Let $0_{\mathrm{T}(E)}=\left(0_{E}, 0_{E}\right)$ and $1_{\mathrm{T}(E)}=\left(1_{E}, 0_{E}\right)$. Then $\mathrm{T}(E)=\left(\mathrm{T}(E), \oplus_{\mathrm{T}(E)}, 0_{\mathrm{T}(E)}, 1_{\mathrm{T}(E)}\right)$ is an effect algebra and the mapping $\varphi: E \rightarrow \mathrm{T}(E)$ given by $\varphi(x)=\left(\widetilde{x}, x \ominus_{E} \widetilde{x}\right)$ is an isomorphism of effect algebras.

Proof: Evidently, $\varphi$ is correctly defined since, for any $x \in E$, we have that $x=\widetilde{x} \oplus_{E}(x \ominus \widetilde{x})=$ $x_{S} \oplus_{E} x_{M}, x_{S} \in \mathbf{S}(E)$ and $x_{M} \in \mathbf{M}(E)$. Hence $\varphi(x)=\left(x_{S}, x_{M}\right) \in \mathbf{S}(E) \times \mathbf{M}(E)$ and $x_{M} \in h\left(x_{S}^{\prime}\right)$. Let us check that $\varphi$ is bijective. Assume first that $x, y \in E$ such that $\varphi(x)=\varphi(y)$. We have $x=\widetilde{x} \oplus_{E}$ $\left(x \ominus_{E} \widetilde{x}\right)=\widetilde{y} \oplus_{E}\left(y \ominus_{E} \widetilde{y}\right)=y$. Hence $\varphi$ is injective. Let $\left(x_{S}, x_{M}\right) \in \mathbf{S}(E) \times \mathbf{M}(E)$ and $x_{M} \in h\left(x_{S}^{\prime}\right)$. This yields that $x=x_{S} \oplus_{E} x_{M}$ exists and evidently by Lemma II.5, (i) $\widetilde{x}=x_{S}$ and $x \ominus_{E} \widetilde{x}=x_{M}$. It follows that $\varphi$ is surjective. Moreover, $\varphi\left(0_{E}\right)=\left(0_{E}, 0_{E}\right)=$ $0_{\mathrm{T}(E)}$ and $\varphi\left(1_{E}\right)=\left(1_{E}, 0_{E}\right)=1_{\mathrm{T}(E)}$. 
Now, let us check that, for all $x, y \in E, x \oplus_{E} y$ is defined iff $\varphi(x) \oplus_{\mathrm{T}(E)} \varphi(y)$ is defined in which case $\varphi\left(x \oplus_{E} y\right)=\varphi(x) \oplus_{\mathrm{T}(E)} \varphi(y)$. For any $x, y, z \in E$ we obtain

$$
\begin{aligned}
& z=x \oplus_{E} y \Longleftrightarrow \\
& z=\left(\widetilde{x} \oplus_{E}\left(x \ominus_{E} \widetilde{x}\right)\right) \oplus_{E}\left(\widetilde{y} \oplus_{E}\left(y \ominus_{E} \widetilde{y}\right)\right) \Longleftrightarrow \\
& z=\left(\widetilde{x} \oplus_{E} \widetilde{y}\right) \oplus_{E}\left(\left(x \ominus_{E} \widetilde{x}\right) \oplus_{E}\left(y \ominus_{E} \widetilde{y}\right)\right) \Longleftrightarrow
\end{aligned}
$$

by Lemma ஹ.1 $(\exists u \in E) u=S\left(x \ominus_{E} \widetilde{x}, y \ominus_{E} \widetilde{y}\right)$ and

$$
\begin{aligned}
z=\left(\widetilde{x} \oplus_{E} \widetilde{y}\right) \oplus_{E} & \left(u \oplus_{E}\left(\left(x \ominus_{E} \widetilde{x}\right) \ominus_{E}\left(u \wedge\left(x \ominus_{E} \widetilde{x}\right)\right)\right) \oplus_{E}\right. \\
& \left.\left(\left(y \ominus_{E} \widetilde{y}\right) \ominus_{E}\left(u \wedge\left(y \ominus_{E} \widetilde{y}\right)\right)\right)\right) \\
\Longleftrightarrow & (\exists u \in E) u=S\left(x \ominus_{E} \widetilde{x}, y \ominus_{E} \widetilde{y}\right) \text { and } \\
z=\left(\widetilde{x} \oplus_{E} \widetilde{y} \oplus_{E} u\right) \oplus_{E} & \left(\left(\left(x \ominus_{E} \widetilde{x}\right) \ominus_{E}\left(u \wedge\left(x \ominus_{E} \widetilde{x}\right)\right)\right) \oplus_{E}\right. \\
& \left.\left(\left(y \ominus_{E} \widetilde{y}\right) \ominus_{E}\left(u \wedge\left(y \ominus_{E} \widetilde{y}\right)\right)\right)\right) \\
\Longleftrightarrow \quad & (\exists u \in E) u=S\left(x \ominus_{E} \widetilde{x}, y \ominus_{E} \widetilde{y}\right) \text { and } \\
z=\left(\widetilde{x} \oplus_{\mathrm{S}(E)} \widetilde{y} \oplus_{\mathrm{S}(E)} u\right) \oplus_{E} & \left(\left(\left(x \ominus_{E} \widetilde{x}\right) \ominus_{\mathrm{M}(E)}\left(u \wedge\left(x \ominus_{E} \widetilde{x}\right)\right)\right) \oplus_{\mathrm{M}(E)}\right. \\
& \left.\left(\left(y \ominus_{E} \widetilde{y}\right) \ominus_{\mathrm{M}(E)}\left(u \wedge\left(y \ominus_{E} \widetilde{y}\right)\right)\right)\right) \\
\Longleftrightarrow & \left(\widetilde{x}, x \ominus_{E} \widetilde{x}\right) \oplus_{\mathrm{T}(E)}\left(\widetilde{y}, y \ominus_{E} \widetilde{y}\right) \text { is defined }
\end{aligned}
$$

and

$$
\begin{aligned}
\varphi(z)=( & \widetilde{x} \oplus_{\mathrm{S}(E)} \widetilde{y} \oplus_{\mathrm{S}(E)} \\
& S\left(x \ominus_{E} \widetilde{x}, y \ominus_{E} \widetilde{y}\right),\left(\left(x \ominus_{E} \widetilde{x}\right) \ominus_{\mathrm{M}(E)}\right. \\
& \left.\left(S\left(x \ominus_{E} \widetilde{x}, y \ominus_{E} \widetilde{y}\right) \wedge\left(x \ominus_{E} \widetilde{x}\right)\right)\right) \oplus_{\mathrm{M}(E)} \\
& \left(\left(y \ominus_{E} \widetilde{y}\right) \ominus_{\mathrm{M}(E)}\right. \\
& \left.\left.\left(S\left(x \ominus_{E} \widetilde{x}, y \ominus_{E} \widetilde{y}\right) \wedge\left(y \ominus_{E} \widetilde{y}\right)\right)\right)\right) \\
= & \left(\widetilde{x}, x \ominus_{E} \widetilde{x}\right) \oplus_{\mathrm{T}(E)}\left(\widetilde{y}, y \ominus_{E} \widetilde{y}\right) \\
= & \varphi(x) \oplus_{\mathrm{T}(E)} \varphi(y) .
\end{aligned}
$$

Altogether, $\mathrm{T}(E)=\left(\mathrm{T}(E), \oplus_{\mathrm{T}(E)}, 0_{\mathrm{T}(E)}, 1_{\mathrm{T}(E)}\right)$ is an effect algebra and the mapping $\varphi: E \rightarrow \mathrm{T}(E)$ is an isomorphism of effect algebras.

The Triple Representation Theorem then follows immediately.
Remark III.3. First, note that any sharply dominating Archimedean atomic lattice effect algebra is a TRT-effect algebra. Namely, any lattice effect algebra is homogeneous. From [12, Theorem 2.10, (iv)] we get that $[\widetilde{x}, x] \subseteq B$, for every block $B$ of $E$ and every $x \in B$. The remaining condition follows from [12, Theorem 2.10, (v)].

Second, any homogeneous orthocomplete effect algebra is a TRT-effect algebra in virtue of Statement II.6. This immediately yields that any complete lattice effect algebra is a TRT-effect algebra.

\section{ACKNOWLEDGEMENTS}

J. Paseka gratefully acknowledges Financial Support of the Ministry of Education of the Czech Republic under the project MSM0021622409 and of Masaryk University under the grant 0964/2009. Both authors acknowledge the support by ESF Project CZ.1.07/2.3.00/20.0051 Algebraic methods in Quantum Logic of the Masaryk University.

\section{REFERENCES}

[1] G. Cattaneo, A unified framework for the algebra of unsharp quantum mechanics, Inter. J. Theor. Phys. 36 (1997), 30853117.

[2] A. Dvurečenskij, S. Pulmannová: New Trends in Quantum Structures, Kluwer Acad. Publ., Dordrecht/Ister Science, Bratislava 2000.

[3] D. J. Foulis, M. K. Bennett, Effect algebras and unsharp quantum logics, Found. Phys. 24 (1994), 1325-1346.

[4] D. J. Foulis, R. Greechie, G. Rütimann, Filters and supports in orthoalgebras, Inter. J. Theor. Phys. 35 (1995), 789-802.

[5] R. J. Greechie, D. J. Foulis, S. Pulmannová, The center of an effect algebra, Order 12 (1995), 91-106.

[6] S. P. Gudder, Sharply dominating effect algebras, Tatra Mt. Math. Publ. 15 (1998), 23-30.

[7] S. P. Gudder, S-dominating effect algebras, Inter. J. Theor. Phys. 37 (1998), 915-923.

[8] G. Jenča, Blocks of homogeneous effect algebras, Bulletin of the Australian Mathematical Society 64 (2001), 81-98.

[9] G. Jenča, Sharp and Meager Elements in Orthocomplete Homogeneous Effect Algebras, Order 27 (2010), 41-61.

[10] G. Jenča, S. Pulmannová, Orthocomplete effect algebras, Proceedings of the American Mathematical Society 131 (2003), 2663-2672 .

[11] F. Kôpka, Compatibility in D-posets, Inter. J. Theor. Phys. 34 (1995), 1525-1531.

[12] J. Niederle, J. Paseka, More about sharp and meager elements in Archimedean atomic lattice effect algebras, Soft Computing, 16 (2012), 109-119.

[13] J. Niederle, J. Paseka, Triple Representation Theorem for orthocomplete homogeneous effect algebras, Algebra Universalis, to appear.

[14] Z. Riečanová, Wu Junde, States on sharply dominating effect algebras, Science in China Series A:Mathematics 51 (2008), 907-914. 